PROCEEDINGS OF THE

AMERICAN MATHEMATICAL SOCIETY

Volume 30, No. 1, September 1971

\title{
ENDOMORPHISMS OF FINITELY PRESENTED MODULES
}

\author{
GABRIEL SABBAGH
}

\begin{abstract}
It is proved that every surjective or injective endomorphism of a finitely presented left module over a right perfect ring is an isomorphism.
\end{abstract}

We will adopt the following conventions: Rings and modules are unitary. Module means left module, ideal means left ideal.

Let $R$ be a ring and $M$ be a finitely generated $R$-module. It is well known that if $R$ is commutative and if $M$ is free, then any two bases of $M$ have the same number of elements. More generally one has ([9], [10]):

(1) If $R$ is commutative, then every surjective endomorphism of $M$ is an isomorphism.

On the other hand, the following has recently been proved [11]:

(2) If $R$ is commutative and of Krull dimension zero, then every injective endomorphism of $M$ is an isomorphism.

In [11] it is suggested that (2) should be valid for rings which are close to being Artinian, as perfect rings. Our main result is:

THEOREM. If $R$ is right perfect and if $M$ is finitely presented, then every injective or surjective endomorphism of $M$ is an isomorphism.

We first need some facts on perfect modules. We recall that a module is said to be perfect if it satisfies the descending chain condition on cyclic submodules. The ring $R$ is said to be right perfect if the (left) $R$-module $R$ is perfect ([2], [3]).

The first of the following statements is Theorem 2 of [3]. The others may easily be derived from it or are immediate.

(3) A perfect module satisfies the descending chain condition on finitely generated submodules.

(4) The quotient of a perfect module by a finitely generated submodule is a perfect module.

(5) Every submodule of a perfect module is a perfect module.

(6) The direct sum of an arbitrary family of perfect modules is a perfect module.

Received by the editors October 19, 1970.

AMS 1969 subject classifications. Primary 1325; Secondary 1340.

Key words and phrases. Injective endomorphism, surjective endomorphism, isomorphism, finitely presented module, perfect ring.

Copyright (c) 1971. American Mathematical Society 
We state as a lemma the following simple consequence of (4) and (6) :

Lemma 1. If $R$ is right perfect, then every finitely presented $R$-module is perfect.

Proposition. Every injective endomorphism of a finitely generated perfect module is an isomorphism.

Proof. Let $X$ be a finitely generated perfect module and let $h$ be an injective endomorphism of $X$. The sequence $\left\langle h^{n}(X), n \geqq 0\right\rangle$ constitutes a descending chain of finitely generated submodules of $X$. By (3) there exists an integer $n$ such that $h^{n}(X)=h^{n+1}(X)$. Since $h$ is injective, one obtains $X=h(X)$.

The next lemma, the first part of which generalizes results of [6] and [7], is anticipated in an interesting although erroneous footnote of [5] and is essentially proved, in rather a different way, in an unpublished dissertation of $\mathrm{G}$. B. Klatt, is of some independent interest.

LEMмa 2. If $R$ is semiperfect, then:

(7) Every projective $R$-module may be written in a unique way as a direct sum of indecomposable cyclic (projective) modules.

(8) Every surjective endomorphism of a finitely generated projective $R$-module is an isomorphism.

Proof. (7): It is well known (and proved in [8]) that if $R$ is semiperfect then the endomorphism ring of every projective indecomposable $R$-module is local. This fact yields the uniqueness part of (7), thanks to Azumaya's version of the Krull-Schmidt theorem [1], and allows us by [12] to reduce the remaining part of (7) to the following: If $R$ is semiperfect, then every free $R$-module $F$ is the direct sum of projective indecomposable cyclic modules. It is clearly enough to prove this in the case where $F$ is the $R$-module $R$ itself. This last step is performed in [8].

(8): It is easy to see that the following assertion, which is an immediate consequence of (7), is equivalent to (8): For every finitely generated projective $R$-module $P$ and every $R$-module $Q$ the $R$ modules $P$ and $P \oplus Q$ are isomorphic only if $Q$ is the 0 module. ${ }^{1}$

We now proceed to the proof of the theorem. We assume that $R$ is a right perfect ring and that $M$ is a finitely presented $R$-module. Let $h$ be an endomorphism of $M$. It follows from Lemma 1 and from the

1 The referee observed that if that assertion holds for the quotient of an arbitrary ring $S$ by its Jacobson radical then it also holds for $S$, which gives another proof of (8). 
proposition that if $h$ is injective then $h$ is an isomorphism. What remains to be shown is that, assuming that $h$ is surjective, $h$ is an isomorphism.

Let $p: P \rightarrow M$ be a projective cover of $M[2]$. By definition $p$ is surjective and, since $M$ is the quotient of a finitely generated projective module, it is easy to see that $P$ is finitely generated. Since $P$ is projective, there exists an endomorphism $g$ of $P$ such that $p \circ g$ $=h \circ p$. Since $(P, p)$ is a projective cover of $M$ and since $p \circ g$ is surjective, it follows that $g$ is surjective. By applying the second part of Lemma 2 we deduce that $g$ is an isomorphism. Let $K$ now be the kernel of $p$. It follows from [4, Lemme 9, p. 37] that $K$ is finitely generated. It is clear that $g$ maps $K$ into $K$. The restriction $g^{\prime}$ of $g$ to $K$ is then an injective endomorphism of $K$. By (5) and Lemma 1 (applied to $P$ which is finitely presented) $K$ is a perfect module. The proposition then implies that $g^{\prime}$ is an isomorphism of $K$. It follows that $h$ is injective. The proof is complete.

We conclude the paper with a proposition inspired by (2).

Proposition. If $R$ is commutative, then every injective pure endomorphism of $M$ is an isomorphism.

Proof. Let $h$ be an injective pure endomorphism of $M$. Our notion of purity is the one introduced by Cohn (see e.g. [4, Ex. 24, p. 66]) and allows us to say that the exact sequence $0 \rightarrow M \stackrel{h}{\rightarrow} M$ remains exact upon tensoring by any cyclic $R$-module. For showing that $h$ is surjective, it suffices by $[4$, Proposition 11, p. 113] to show that for every maximal ideal $\mathfrak{N}$ of $R$ the homomorphism $h_{\Re}: M / \mathfrak{M} M$ $\rightarrow M / \mathfrak{T} M$ induced by $h$ is surjective. The modules $R / \mathfrak{m} \otimes M$ and $M / \mathfrak{M} M$ are canonically isomorphic and we may identify $h_{\Re}$ with $1 \otimes h: R / \mathfrak{M} \otimes M \rightarrow R / \mathfrak{M} \otimes$. Hence $h_{\Re}$ is injective. Since $M$ is finitely generated, the $R / \mathfrak{m}$-vector space $M / \mathfrak{T} M$ is of finite dimension. It follows that $h_{\mathfrak{M}}$ is surjective and the proof is complete.

Two remarks are in order:

(a) Let $N$ denote the two-sided ideal generated by the nilpotent elements of $R$. If $R$ is commutative, $R$ is of Krull dimension zero if and only if the ring $R / N$ is a von Neumann regular ring [4, Ex. 16, p. 173]. On the other hand, if $S$ is a von Neumann regular ring, every $S$-module is a pure submodule of every $S$-module containing it. The connection between (2) and the previous proposition is now clear.

(b) The assumption of the commutativity of $R$ cannot be dispensed with in the previous proposition. Indeed, let $V$ denote a vector space of infinite dimension over an arbitrary field $K$. Let us take for $R$ the ring of all $K$-endomorphisms of $V$. It is easy to see (and well known) 
that there exists an isomorphism $f$ of the $R$-module $R \oplus R$ onto the $R$-module $R$. Let $j$ denote the embedding: $x \rightarrow(x, 0)$ of $R$ into $R \oplus R$. It is clear that the injective endomorphism $f \circ j$ of $R$ is pure and is not an isomorphism. Note that in that example $R$ is a von Neumann regular ring.

\section{REFERENCES}

1. G. Azumaya, Corrections and supplementaries to my paper concerning KrullRemak-Schmidt's theorem. Nagoya Math. J. 1 (1950), 117-124. MR 12, 314.

2. H. Bass, Finitistic dimension and a homological generalization of semi-primary rings, Trans. Amer. Math. Soc. 95 (1960), 466-488. MR 28 \#1212.

3. J. E. Björk, Rings satisfying a minimum condition on principal ideals, J. Reine Angew. Math. 236 (1969), 112-119. MR 40 \#1419.

4. N. Bourbaki, Algèbre commutative. Chaps. 1, 2, Actualités Sci. Indust., no. 1290, Hermann, Paris, 1961. MR 36 \#146.

5. P. M. Cohn, Hereditary local rings, Nagoya Math. J. 27 (1966), 223-230. MR $33 \# 5663$.

6. S. Eilenberg, Homological dimension and syzygies, Ann. of Math. (2) 64 (1956), 328-336. MR 18, 558.

7. I. Kaplansky, Projective modules, Ann. of Math (2) 68 (1958), 372-377. MR 20 \#6453.

8. J. Lambek, Lectures on rings and modules, Blaisdell, Toronto, 1966. MR 34 \#5857. \#5679.

9. J. R. Strooker, Lifting projectives, Nagoya Math. J. 27 (1966), 747-751. MR 33

10. W. V. Vasconcelos, On finitely generated flat modules, Trans. Amer. Math. Soc. 138 (1969), 505-512. MR 39 \#199.

11. - Injective endomorphisms of finitely generated modules, Proc. Amer. Math. Soc. 25 (1970), 900-901.

12. R. B. Warfield, Jr., A Krull-Schmidt theorem for infinite sums of modules, Proc. Amer. Math. Soc. 22 (1969), 460-465. MR 39 \#4213.

1 square François Couperin, 92-Antony, France 